\title{
The Effects of Exergame on Postural Control in Individuals with Ataxia: a Rater-Blinded, Randomized Controlled, Cross-over Study
}

\author{
Ender Ayvat ${ }^{1}\left(\mathbb{D} \cdot\right.$ Özge Onursal Kılınç $^{1} \cdot$ Fatma Ayvat $^{1} \cdot$ Cevher Savcun Demirci ${ }^{2}$. Sibel Aksu Yıldırım ${ }^{1}$. \\ Oğuzhan Kurşun ${ }^{3} \cdot$ Muhammed Kılınç $^{1}$
}

Accepted: 2 May 2021 / Published online: 11 May 2021

(c) The Author(s), under exclusive licence to Springer Science+Business Media, LLC, part of Springer Nature 2021

\begin{abstract}
Exergame trainings might have therapeutic value in ataxic patients. The aim of this study was to investigate the effect of exergame training with an exercise program on postural control by comparing it with traditional balance and coordination exercise program. Nineteen patients were randomly allocated to two groups. In the first group, exergame training and an exercise program (EEP) were applied together for the first 8 weeks; after 10 weeks washout, a conventional exercise program (CEP) was applied for the second 8 weeks. In the second group, the CEP was applied first followed by the EEP. Outcome measures were Limits of Stability test (LoS), International Classification Ataxia Ratio Scale (ICARS), Berg Balance Scale (BBS), and Timed-Up and Go test with a cognitive task (TUG-C), Reactive postural control and sensory orientation subscales of the Mini-BESTest. Seventeen patients (mean age \pm SD, $32.53 \pm 11.07$ years) completed the study. ICARS, BBS scores improved only after EEP $(p<0.05)$. While there was no change in the RT and MVL parameters of the LoS test after EEP, the MXE, EPE, and DCL parameters improved significantly $(p<0.05)$. The MXE and MVL parameters of LoS improved after CEP $(p<0.05)$. There were no significant improvements in the Mini-BESTest's reactive postural control and sensory orientation subscale scores after both EEP and CEP $(p>0.05)$. The results of the present study demonstrated that exergame training can be used as a complementary training option in physiotherapy to improve postural control in patients with ataxia. ClinicalTrial.gov Identifier: NCT03607058
\end{abstract}

Keywords Exergames $\cdot$ Exercise therapy $\cdot$ Ataxia $\cdot$ Postural control $\cdot$ Rehabilitation $\cdot$ Virtual reality

Ender Ayvat

ender.ayvat@gmail.com

Özge Onursal Kılınç

ozgeonursal@hotmail.com

Fatma Ayvat

fatma.avcu@hacettepe.edu.tr

Cevher Savcun Demirci

cevhersavcun@hotmail.com

Sibel Aksu Yıldırım

sibelaksu@yahoo.com

Oğuzhan Kurşun

ozikursun@hotmail.com

Muhammed Kılınç

muhammedkilinc@yahoo.com

1 Faculty of Physical Therapy and Rehabilitation, Hacettepe University, 06100 Ankara, Turkey

2 Faculty of Health Sciences, Department of Physiotherapy and Rehabilitation, Balıkesir University, Balıkesir, Turkey

3 Neurology Clinic, Ministry of Health Ankara City Hospital, Ankara, Turkey

\section{Introduction}

Ataxia causes symptoms that affect basic functions such as speech, walking, balance, and eye movements apart from disorders such as, motor coordination disorders, dysmetria, dysdiadokinesia, and tremor [1]. Disorders in the cerebellum (multiple sclerosis, stroke), spinal cord pathologies, disorders affecting the peripheral sensory system, or their combinations may result in ataxia [2,3]. There are no pharmacological treatments that are effective in reducing these symptoms and/or ataxia progression. On the other hand, rehabilitation approaches play an important role in controlling ataxia progression and improving patients' functions [4].

Rehabilitation approaches play an important role in eliminating balance and gait disorders, reducing fear of falling, increasing social participation, and independence in daily life activities. In general, static-dynamic balance and walking exercises, coordination exercises, and exercises to 
improve the proprioceptive sense are used in ataxia rehabilitation [5]. For the success of such rehabilitation programs, it is important to perform exercises with intensive repetition, purposefulness, and high patient participation and motivation to support the motor learning process $[6,7]$.

These applications require one-to-one interaction with the therapist, are costly for patients, and become monotonous for patients over time, as they continue for a long time. Therefore, the application of different physiotherapy options in progressive neurological diseases due to the chronic and long course of the disease is important in terms of ensuring patient participation and motivation. One of the most widely used alternative treatments today is exergame trainings/active video game-based therapies [8-10].

Active video game-based therapy reduces the boredom of the rehabilitation process, increases the motivation of the patients, provide direct feedback, and allow dual-task training [11]. These applications can be used in the early, middle, and advanced stages of ataxia, but well-structured studies are needed in terms of the level of evidence, game selection, and suitability of the control group [12].

In a 2014 reviewer of therapies used to treat patients with ataxia, Synofzik \& Ilg [12] stated that exergame-based training should not replace physiotherapy and it might rather serve to complement physiotherapy-based programs by helping patients to achieve the required training intensity even over a long training period. Furthermore, in another review in 2020, these implications were supported, and the researchers stated that exergaming is not superior to the conventional balance and coordination training in improving the balance [13]. Previous studies investigated the effects of exergaming on balance, the severity of ataxia, gait, and some participation parameters in ataxic patients [14-16]. However, other components of postural control have been ignored so far and exergame trainings were not used to complement exercise programs. The aim of this study was to examine the effect of exergame training with an exercise program on postural control by comparing it with conventional balance and coordination exercise program.

\section{Materials and Methods}

\section{Participants}

This study was conducted in the Faculty of Physical Therapy and Rehabilitation, Hacettepe University. It was approved by the Ethics Committee of the Hacettepe University (KA-17049).

The inclusion criteria had four parameters:

1. Being diagnosed with cerebellar ataxia by a neurologist
2. Walking independently (without any help or assistive device)

3. Mini-Mental State Examination [17] score $\geq 24$ points

4. Having an Expanded Disability Status Scale [18] cerebellar system score $\geq 3$ for ataxic multiple sclerosis patients

Exclusion criteria for the study included six parameters:

1. Predominantly vestibular, sensory ataxia, and muscle weakness

2. Systemic diseases and cognitive impairment

3. Communication problems

4. Other orthopedic and neurological problems that may affect postural control

5. Increased muscle tone of extremity muscles (Modified Ashworth Scale score $\geq 2$ )

6. Having an Expanded Disability Status Scale pyramidal functional system score $>3$ for ataxic multiple sclerosis patients

Among the patients who were referred to the outpatient clinic from different hospitals in Ankara, those who met the inclusion criteria were selected. The patients who agreed to participate in the study were informed in detail about the study and gave written informed consent.

\section{Design}

The study was designed as a randomized controlled, assessor-blinded, cross-over trial. Randomization was performed using a computer program and patients were randomly allocated to two groups via the program. In the first group, exergame training with an exercise program (EEP) was applied together for the first 8 weeks; after 10 weeks washout period, conventional exercise program (CEP) was applied for the second 8 weeks. In the second group, the CEP was applied for the first 8 weeks, and after 10 weeks washout period, EEP was applied for the second 8 weeks. The flow chart of the study is shown in Fig. 1.

\section{Outcome Measures}

The assessments were applied four times before and after both treatments by the same physiotherapist who was blind to the study. Demographic information such as age, gender, body weight, height, and type of diagnosis was recorded before the first assessment. To examine all sources of postural control (sensory strategies, movement strategies, biomechanical constraints, orientation in space, control of dynamics, and cognitive processing) within the scope of the study, the evaluations were determined taking these sources into consideration and applied to the participants [19]. Short breaks (approximately 


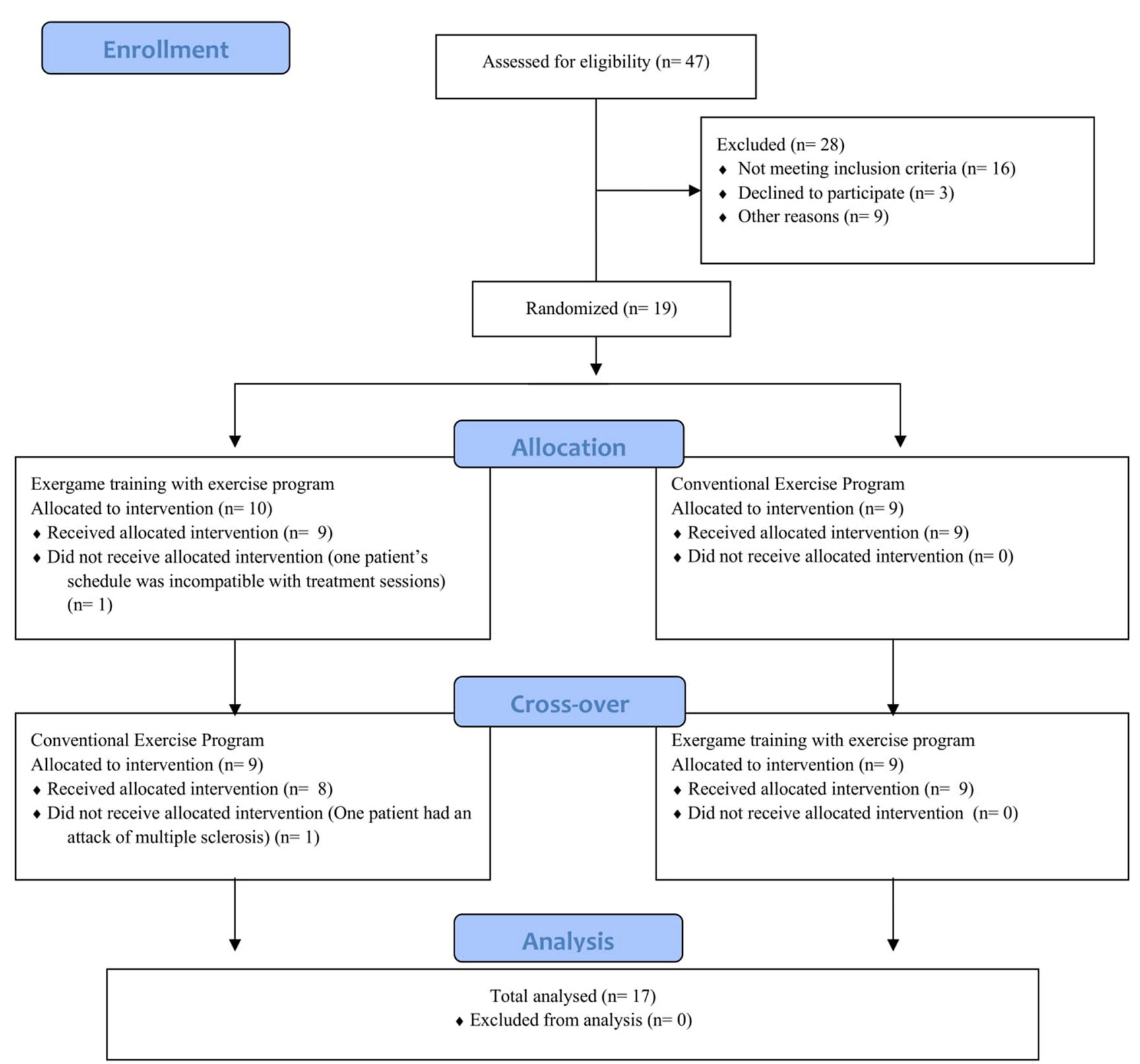

Fig. 1 Flow chart of the study

5-10 $\mathrm{min}$ ) were given between posturography and other clinical assessments to prevent fatigue. Also, these evaluations were applied randomly to prevent the learning effect.

\section{Severity of Ataxia}

The International Cooperative Ataxia Rating Scale (ICARS) was used to assess the severity of ataxia, consists of four subscales: posture and gait disturbances ( 7 items; 34 points), kinetic functions ( 7 items; 52 points), speech disorders ( 2 items; 8 points), and oculomotor disorders ( 3 items; 6 points). The total scores range from 0 (normal) to 100 (most severe ataxia) [20]. The ICARS was used to assess the "control of dynamics" component of the postural control.

\section{Functional Mobility with Dual Task}

The Timed-Up and Go test (TUG) was used to assess the functional mobility of the individuals. Individuals were asked to get up from the chair and walk $3 \mathrm{~m}$ away and then go back to the same distance and sit in the chair again; the time was measured with a chronometer. The average time was calculated by repeating three times. The test was then repeated by adding a cognitive additional task. The cognitive task was applied in the form of a 3-by-3 count back from 100 [21]. 
TUG tests were used to assess the "cognitive processing" component of the postural control.

\section{Performance-based Balance}

The performance-based balances of the individuals were assessed with the Berg Balance Scale (BBS). The BBS is a scale that includes scoring between 0 (not applicable) and 4 (normal performance) for the performance of 14 different tasks. This scale is evaluated over 56 points, and scores from 0 to 20 indicate the loss of balance, scores from 21 to 40 indicate that balance is acceptable, and scores from 41 to 56 indicate good balance [22]. The BBS was used to assess the "orientation in space" component of the postural control.

\section{Limits of Stability Test}

The Limits of Stability test of computerized static posturography (Neurocom Balance Master System Inc., Clackamas, OR) evaluates the ability to control the movement of the gravity to center over the support surface. The voluntary control of the gravity center is achieved by asking the patient to shift their weight in 8 different directions. In each direction, maximum excursion (MXE), the reaction time (RT), directional control (DCL), endpoint excursion (EPE), and movement velocity (MVL) parameters were evaluated [23]. The LoS was used to assess the "biomechanical constraints" component of the postural control.

\section{Sensory and Movement Strategies}

The Mini-BESTest is a 14-item test that focuses on different components of postural control and has 4 headings; anticipatory transitions, reactive postural control, sensory orientation, and dynamic gait. Each item is scored from 0 to 2 ; a score of 0 indicates that a person is unable to perform the task while a score of 2 is normal. The best score is the maximum amount of points, being 28 [24]. Reactive postural control and sensory orientation subscales were used to assess "movement strategies" and "sensory strategies" components of the postural control, respectively.

\section{Interventions}

The common goals of both treatment protocols applied within the scope of the study were determined as increasing trunk and proximal stabilization, improving balance and postural reactions against external stimuli and gravity, improving the functions of the upper extremities, and functional walking, increasing independence. The participants were randomized into two groups and entered into the physiotherapy program for 16 weeks ( 8 weeks $\times 2$ ): 3 days a week for $1 \mathrm{~h}$ per day.

\section{Exergame Training with Exercise Program}

In the first phase of the study, commercially available Microsoft Kinect games were personally monitored and applied by four physiotherapists who are experts in the field of neurological rehabilitation. Then, games were analyzed in accordance with the neurodevelopmental treatment principles and targeted exercise gains. As a result of this analysis, five games that could meet our treatment goals in patients with ataxia were selected for the study (Table 1).

In the EEP, one treatment session of the cases was created from 40 min of exergame application and $20 \mathrm{~min}$ of exercise program. In the exergame application, the subjects played each game in two repetitions in a single session, accompanied by a physiotherapist. Each game lasted approximately 3-4 min, and during game breaks, subjects were seated in a chair for resting. The exercises applied to the cases in the EEP were formed from the following approaches:

- Deep massage to improve the underfoot sense

- Mobilization of the foot and ankle joints

- Weight transfer and stabilization training in supine, crawling and kneeling positions (with sensory stimulation techniques)

- Frenkel coordination exercises (with sensory stimulation techniques)

The difficulty level of the games was determined by the individual performance of the cases and progress; based on the scores achieved in games, improvements in postural stabilization, and feedback from the cases.

\section{Conventional Exercise Program}

One treatment session of the cases in the exercise protocol was created from a 60 -min exercise program. In this protocol, the following exercise program was applied to the subjects:

- Deep massage to improve the underfoot sense

- Mobilization of the foot and ankle joints

- MAT activities (approximation and dynamic balance training from proprioceptive neuromuscular facilitation techniques in supine, crawling, and kneeling positions)

- Static and dynamic balance exercises (rhythmic stabilization and stabilizing reversal from proprioceptive neuromuscular facilitation techniques in sitting and standing positions)

- Frenkel coordination exercises (with sensory stimulation techniques)

- Weight shifting in standing position (on changing support base and different grounds) 
Table 1 Analysis of Kinect games for therapeutic purposes

\begin{tabular}{|c|c|c|}
\hline Games & Definitions & Targeted exercise gains \\
\hline Joy Ride & $\begin{array}{l}\text { Car simulation. The player stretches his arms forward as } \\
\text { if holding the invisible steering wheel, directing the car } \\
\text { with body movements. The player accelerates the car } \\
\text { with the forward movement of his/her trunk }\end{array}$ & \multirow{5}{*}{$\begin{array}{l}\text {-Weight shifting and multidirectional steps, } \\
\text {-Coordination of voluntary limb movements, } \\
\text {-Balanced coordination, } \\
\text {-Trunk\&proximal extremity stabilization, } \\
\text {-Postural balance and correction reactions, } \\
\text {-Selective body movements, } \\
\text {-Hand/eye coordination, } \\
\text {-Reaction time, } \\
\text {-Proprioceptive stimulation, } \\
\text {-Plyometric control, } \\
\text {-Concentric and eccentric control of the lower extremity } \\
\text { postural muscles, } \\
\text {-Increasing limb and trunk muscle strength and endur- } \\
\text { ance, } \\
\text {-Vestibular stimulation, } \\
\text {-Postural preparation activities }\end{array}$} \\
\hline Adventures River Rush & $\begin{array}{l}\text { Raft simulation. The player provides raft navigation by } \\
\text { leaning forward and sideways and also collects bonuses } \\
\text { using his/her upper limbs. The player avoids obstacles } \\
\text { by jumping and sideway steps }\end{array}$ & \\
\hline Adventures Rally Ball & $\begin{array}{l}\text { The player hits oncoming balls and smashes blocks using } \\
\text { the } 4 \text { limbs and head, when more than one ball comes, } \\
\text { coordinated movement of the extremities is required. } \\
\text { The player tries to meet the balls by taking sideways } \\
\text { steps }\end{array}$ & \\
\hline Kinect sports skiing & $\begin{array}{l}\text { Ski simulation. The player holds the invisible batons and } \\
\text { directs the avatar with body movements. The player } \\
\text { uses his/her upper extremities to accelerate. There are } \\
\text { sections on the route that include crouching and jump- } \\
\text { ing }\end{array}$ & \\
\hline Adventures 20,000 Leaks & $\begin{array}{l}\text { The player is underwater in a glass cube. Using his/her } \\
\text { arms, legs, and head he/she tries to seal the leaks in the } \\
\text { glass }\end{array}$ & \\
\hline
\end{tabular}

- Walking training (walking on two narrow lines, tandem walking, soldier walking, walking on different grounds, stopping and turning with sudden commands)

The difficulty level of the exercises was determined according to the individual performance of the cases and the exercise programs were developed by the physiotherapist in line with the following main principles;

- Activities for coordination of distal segments when proximal tonus and stabilization responses develop

- From exercises involving conscious movements to exercises involving automatic activities

- From simple exercises to complex exercises covering different parts of the body

- From exercises done on stable surfaces to exercises done on unstable surfaces (in different sensory stimulation and environmental conditions)

As a result of the symptoms of fatigue during trainings, the session was interrupted and the patient was rested, and the sessions continued after the change of complaints.

\section{Statistical Analysis}

Statistical analyses were performed using the SPSS software package (version 20.0, SPSS Inc., Chicago, IL). The quantitative data were expressed as median (25-75\% interquartile range) (median (IQR)). The normal distribution of the obtained data was evaluated visually (histogram and probability plots) and by using the Shapiro-Wilk test. Nonparametric tests were used because the data obtained were not normally distributed.

Since the study was planned as a cross-over design and the data did not show normal distribution, first of all, the Mann-Whitney $U$ test was used to compare baseline assessments of two groups and then the difference between the period effects was evaluated with the Mann-Whitney $U$ test. Wilcoxon two-tailed paired test was used to compare the efficacy of treatment programs, between baseline and after treatments; the level of significance was set at $p<0.05$.

\section{Results}

The study began with 19 patients. A patient from group 1 left the study because his schedule was incompatible with treatment sessions. And also a patient from group 2 left the study because of a multiple sclerosis attack. As such, data of 17 patients were included in the analyses. The demographic information of the patients was given in Table 2 .

There was no statistically significant difference in the baseline assessment scores of two groups $(p>0.05)$ (Table 3). In order to examine the effect of applying either of the two exercise approaches before or after, the period effect was examined. At the end of the analysis, there was no statistically significant difference between group 1 and group 2 in all of the parameters evaluated $(p>0.05)$ (Table 4$)$.

In the study, since all parameters had both no difference at baseline assessments and no period effect, the data of the 
Table 2 Demographics of patients

\begin{tabular}{lllll}
\hline & $\begin{array}{l}\text { Group 1 } \\
(n=8) \\
\text { Median (IQR) }\end{array}$ & $\begin{array}{l}\text { Group 2 } \\
(n=9) \\
\text { Median (IQR) }\end{array}$ & $Z$ & $p$ \\
\hline Age (year) & $26(21.5-52)$ & $31(26-37)$ & 0.501 & 0.617 \\
Body mass index $\left(\mathrm{kg} / \mathrm{m}^{2}\right)$ & $22.02(20.06-27.51)$ & $24.38(23.14-27.85)$ & 0.857 & 0.391 \\
Duration of disease (month) & $138(100.5-150)$ & $84(72-144)$ & -0.863 & 0.388 \\
Gender (female/male) & $5 / 3$ & $6 / 3$ & & \\
Diagnoses & 5 Multiple sclerosis, 3 & 7 Multiple sclerosis, 2 & & \\
& Spinocerebellar ataxia & Spinocerebellar ataxia & & \\
\hline
\end{tabular}

"p $<0.05$ Mann-Whitney $U$ test groups were combined and subsequent analyses were done accordingly.

ICARS, BBS scores improved only after EEP $(p<0.05)$ (Table 5). While there was no change in the RT and MVL parameters of the LoS test after EEP, the MXE, EPE, and DCL parameters improved significantly $(p<0.05)$. The MXE and MVL parameters of LoS improved after CEP $(p<0.05)$. There were no significant improvements in the mini-BESTest's reactive postural control and sensory orientation subscale scores after both EEP and CEP $(p>0.05)$ (Table 5).

There was a significant improvement in TUG with additional cognitive tasks after EEP $(p<0.05)$. A cognitive task-based difference [(TUG-C)-(TUG)] was calculated to determine the burden of the cognitive task in the TUG with an additional cognitive task. The increase in time that was caused by the additional cognitive task decreased only after EEP $(p<0.05)$ (Table 5).
Power analysis (Gpower 3.0.10 program) calculated according to MXE parameter of LoS test results reached $83.7 \%$ power (effect size $\mathrm{dz}=0.78$, critical $t=2.12$, $\mathrm{Df}=15.23)$ in the $95 \%$ confidence interval.

\section{Discussion}

In the present study, we aimed to examine the effectiveness of individualized exergame training with an exercise program on postural control parameters in ataxic patients in comparison to conventional exercise training consisting of balance and coordination exercises. Our results demonstrated a significant reduction of ataxia severity, improvements in performance based balance, stability limits, and cognitive processing after EEP. On the contrary, limited improvements were obtained that did not reach statistical significance after CEP.
Table 3 Baseline scores of groups

\begin{tabular}{lllrl}
\hline & $\begin{array}{l}\text { Group 1 } \\
(n=8) \\
\text { Median (IQR) }\end{array}$ & $\begin{array}{l}\text { Group 2 } \\
(n=9) \\
\text { Median (IQR) }\end{array}$ & $Z$ & $p$ \\
\hline ICARS (0-100) & $9(3.75-15)$ & $13(7-18)$ & 0.936 & 0.349 \\
BBS (0-56) & $53.5(50.75-55.25)$ & $51(48-52)$ & -1.801 & 0.072 \\
LoS_MXE & $87.31(76.88-92.44)$ & $81.88(74-88.75)$ & -1.000 & 0.317 \\
LoS_RT & $0.90(0.71-1.40)$ & $0.77(0.70-1.30)$ & -0.571 & 0.568 \\
LoS_MVL & $3.79(2.74-4.41)$ & $3.61(2.18-5.60)$ & -0.429 & 0.668 \\
LoS_EPE & $71.69(54.75-81.34)$ & $62.12(55.38-69.88)$ & -0.930 & 0.352 \\
LoS_DCL & $76.38(65.84-78.84)$ & $61.75(52.63-79)$ & -1.143 & 0.253 \\
MB-RPS & $6(4.75-6)$ & $6(3-6)$ & -0.736 & 0.462 \\
MB-SO & $4(4-6)$ & $4(3-5)$ & -0.938 & 0.348 \\
TUG & $6.98(6.14-8.31)$ & $8.47(6.39-8.78)$ & 1.000 & 0.317 \\
TUG-C & $8.01(7.13-9.95)$ & $8.83(8.21-9.84)$ & 0.571 & 0.568 \\
(TUG-C)-(TUG) & $0.49(0.24-2.18)$ & $0.98(0.23-1.67)$ & 0.286 & 0.775 \\
\hline
\end{tabular}

${ }^{*} p<0.05$ Mann-Whitney $U$ test

ICARS, International Cooperative Ataxia Rating Scale; BBS, Berg Balance Scale; $L o S \_M X E$, Limits of Stability test maximum excursion; $L O S \_R T$, Limits of Stability test reaction time; $L o S \_M V L$, Limits of Stability test movement velocity; $L O S \_E P E$, Limits of Stability test endpoint excursion; $L O S \_D C L$, Limits of Stability test directional control; $M B-R P S$, Mini-BESTest reactive postural control; $M B-S O$, Mini-BESTest sensory orientation; $T U G$, Timed-Up and Go test; $T U G-C$, Timed-Up and Go test with cognitive task 
Table 4 Comparisons of period effects

\begin{tabular}{lllll}
\hline & $\begin{array}{l}\text { Group 1 } \\
(n=8) \\
\text { Median (IQR) }\end{array}$ & $\begin{array}{l}\text { Group 2 } \\
(n=9) \\
\text { Median (IQR) }\end{array}$ & $Z$ & $p$ \\
\hline ICARS $(0-100)$ & $-2((-3.5)-(-0.75))$ & $-2((-4)-(-1))$ & -0.292 & 0.770 \\
BBS (0-56) & $1.5(0-2.75)$ & $2(1-2)$ & 0.523 & 0.601 \\
LoS_MXE & $6.63((-7.56)-15.91))$ & $5.88(1.88-17.88)$ & 0.429 & 0.668 \\
LoS_RT & $-0.1((-0.46)-(0.3))$ & $-0.14((-0.34)-(0.01))$ & -0.143 & 0.886 \\
LoS_MVL & $1.90(0.43-2.73)$ & $0.54((-0.49)-(1.16))$ & -1.429 & 0.153 \\
LoS_EPE & $10.88((-12.06)-(29.88))$ & $10.25(9.13-18)$ & 0.358 & 0.721 \\
LoS_DCL & $-0.75((-3.66)-(6.59))$ & $5.00((-0.75)-(20.87))$ & 1.000 & 0.317 \\
MB-RPS & $0(0-1.25)$ & $0(0-2)$ & 0.490 & 0.624 \\
MB-SO & $1.50(0-2)$ & $1(0-2)$ & -0.076 & 0.940 \\
TUG & $-0.53((-1.22)-(0.01))$ & $-0.39((-1.42)-(0.04))$ & 0.143 & 0.886 \\
TUG-C & $-0.79((-1.28)-(-0.46))$ & $-0.90((-1.51)-(-0.66))$ & -0.571 & 0.568 \\
(TUG-C)-(TUG) & $-0.23\left((-0.99)-(0.42) \_\right.$ & $-0.25((-0.77)-(0.05))$ & 0.072 & 0.943 \\
\hline
\end{tabular}

${ }^{*} p<0.05$ Mann-Whitney $U$ test

ICARS, International Cooperative Ataxia Rating Scale; BBS, Berg Balance Scale; $L o S \_M X E$, Limits of Stability test maximum excursion; $L o S \_R T$, Limits of Stability test reaction time; $L o S \_M V L$, Limits of Stability test movement velocity; $L O S \_E P E$, Limits of Stability test endpoint excursion; $L O S \_D C L$, Limits of Stability test directional control; $M B-R P S$, Mini-BESTest reactive postural control; $M B$-SO, Mini-BESTest sensory orientation; $T U G$, Timed-Up and Go test; TUG-C, Timed-Up and Go test with cognitive task
The LoS test was used to evaluate the "biomechanical constraints" that affect the selection of movement strategies required for postural control. In the previous studies, only one study used the test to investigate the effectiveness of exergames in ataxic patients. Wang et al. applied exergames with warm-up and cool-down stretching exercises for 4 weeks/12 sessions and found no improvement in LoS [25]. In the present study, improvements were observed in MXE,

Table 5 Results of outcome measures

\begin{tabular}{|c|c|c|c|c|c|c|c|c|}
\hline & \multicolumn{4}{|c|}{$\begin{array}{l}\text { Conventional exercise program } \\
(n: 17)\end{array}$} & \multicolumn{4}{|c|}{$\begin{array}{l}\text { Exergame training with exercise program } \\
(n: 17)\end{array}$} \\
\hline & $\begin{array}{l}\text { Pre-treatment } \\
\text { Median (IQR) }\end{array}$ & $\begin{array}{l}\text { Post-treatment } \\
\text { Median (IQR) }\end{array}$ & $Z$ & $p$ & $\begin{array}{l}\text { Pre-treatment } \\
\text { Median (IQR) }\end{array}$ & $\begin{array}{l}\text { Post-treatment } \\
\text { Median (IQR) }\end{array}$ & $Z$ & $\mathrm{p}$ \\
\hline $\begin{array}{l}\text { ICARS } \\
(0-100)\end{array}$ & $11(3.5-14.5)$ & $11(3.5-13)$ & -1.329 & 0.184 & $12(4.5-16)$ & $11(2.5-13)$ & -2.444 & $0.015^{*}$ \\
\hline $\begin{array}{l}\text { BBS } \\
(0-56)\end{array}$ & $53(52-54)$ & $53(52-55)$ & 0.905 & 0.366 & $52(51-54)$ & $53(52-55.5)$ & 2.358 & $0.018^{*}$ \\
\hline LoS_MXE & 77.87 (70.62-92.50) & $92.75(89.00-96.37)$ & 2.341 & $0.019 *$ & $84(74.44-88.88)$ & $92.50(84.44-95.63)$ & 2.622 & $0.009 *$ \\
\hline LoS_RT & $0.83(0.74-1.02)$ & $0.82(0.71-0.99)$ & -0.734 & 0.463 & $0.87(0.70-1.09)$ & $0.79(0.75-0.97)$ & -0.804 & 0.422 \\
\hline LoS_MVL & $3.28(2.73-4.38)$ & $4.70(3.25-5.31)$ & 2.970 & $0.003 *$ & $4.60(3.34-5.22)$ & $4.38(3.24-5.77)$ & 0.665 & 0.506 \\
\hline LoS_EPE & $70.25(54.63-78.13)$ & $75.75(66.25-81.94)$ & 1.223 & 0.221 & $67.25(60.63-74.25)$ & $72(65.56-82.63)$ & 2.132 & $0.033^{*}$ \\
\hline LoS_DCL & 75 (70.63-78.94) & $69.38(58.44-79.38)$ & -1.293 & 0.196 & $64.25(56.75-77)$ & $74.5(70-78.25)$ & 2.132 & $0.033^{*}$ \\
\hline MB-RPS & $6(5.5-6)$ & $6(5.5-6)$ & 0.577 & 0.564 & $6(4.5-6)$ & $6(6-6)$ & 1.857 & 0.063 \\
\hline MB-SO & $5(4-6)$ & $6(5-6)$ & 1.890 & 0.059 & $5(4-6)$ & $6(5-6)$ & 1.807 & 0.071 \\
\hline TUG & 7.17 (6.13-8.47) & $6.77(6.02-8.31)$ & -1.433 & 0.152 & $6.77(6.19-8.69)$ & $6.48(6.05-8.47)$ & -1.538 & 0.124 \\
\hline TUG-C & $8.11(7.13-9.19)$ & $8.17(6.78-8.96)$ & -1.642 & 0.101 & $8.83(6.97-9.82)$ & $8.11(6.35-8.85)$ & -2.971 & $0.003^{*}$ \\
\hline (TUG-C)-(TUG) & $0.41(0.23-1.30)$ & $0.31(0.11-1.44)$ & -1.363 & 0.173 & $0.98(0.24-1.78)$ & $0.41(0.12-1.58)$ & -2.040 & $0.041^{*}$ \\
\hline
\end{tabular}

${ }^{*} p<0.05$ Wilcoxon two-tailed paired test

ICARS, International Cooperative Ataxia Rating Scale; BBS, Berg Balance Scale; LoS_MXE, Limits of Stability test maximum excursion; LoS_ $R T$, Limits of Stability test reaction time; $L o S \_M V L$, Limits of Stability test movement velocity; LoS_EPE, Limits of Stability test endpoint excursion; $L O S \_D C L$, Limits of Stability test directional control; $M B-R P S$, Mini-BESTest reactive postural control; $M B-S O$, Mini-BESTest sensory orientation; TUG, Timed-Up and Go test; TUG-C, Timed-Up and Go test with cognitive task 
EPE, and DCL after EEP. We thought that the application of exergames and exercise program together and 8 weeks training duration allowed the patients to gain more than Wang's study. Also, in the present study, stimulating slower and more controlled movements was aimed because of the disorder of voluntary co-contractions of agonist/antagonist postural muscles and affected corrective movements involving the whole body in ataxia [26]. Therefore, no change in the velocity and the reaction time parameters after treatment can be considered the desired result.

In previous studies evaluating the efficacy of exergames in ataxia, the most commonly used outcome was the severity of ataxia assessed as a component of the "control of dynamics" in the study. According to previous studies, the severity of ataxia decreased after 4 weeks, 8 weeks, and 12 weeks exergame training $[14,16,25]$. And the results of the present study in line with these studies. These findings imply that exergame interventions may be able to postpone disease progression, especially the ataxia. Also, based on the results from those related studies, it seems that the more intensive training provides the greater reduction in the severity of ataxia.

Another result of our study was the performance-based balance selected for the "spatial orientation" component of postural control improved only after EEP. We found average decreases in BBS scores of 1.2 points after exergame training and our findings also corroborate the results of a prior study evaluating balance based on performance [15]. Adding exergames to traditional exercise programs leads to significant improvements in balance control by stimulating motor coordination and input-output integration underlying maintenance of balance $[15,27]$.

The performance of postural tasks can be impaired by the secondary cognitive tasks because postural control and other cognitive tasks share cognitive resources [28]. Exergaming could be considered a dual-task strategy to train persons to automate the motor task, allowing the allocation of more resources to other tasks [29]. Dual-task execution requires prioritization of a task, i.e., to hierarchically select the relevant task [30]. The results of the present study also revealed improvements in the cognitive-TUG test that support these theoretical inferences.

The present study has a noteworthy limitation; posttreatment follow-up data to reveal whether the benefits of the exergame training are maintained long term. Other limitations of this study include heterogeneity of the included ataxia subjects, small sample size. Future studies specifically tackling these limitations are warranted to confirm the effects observed in the present study. Several patients (using many drugs that suppress the autoimmune system) naturally refused to participate in our study during the COVID19 days. Also taking into account the impossibility to foresee the progress of this pandemic, the study had to be completed earlier than the previously scheduled time. Nonetheless, we deemed our current results worthwhile to discuss.

In a 2020 reviewer of the use of gaming technologies in ataxia, Lacorte and colleagues [31] stated that future studies should be a blind assessor and randomized controlled trial. Moreover, Lanza et al. indicated that exergame-based training can enhance the effect of conventional physiotherapy and also professional expertise is required for the selection of the appropriate exergame based on the subject's coordinative ability and level of impairment. The present study eliminates the lack stated in these two important studies.

\section{Conclusions}

The results of this study provide significant knowledge for the effectiveness of combined exergame and exercise training in patients with ataxia. The addition of exergame training to the traditional exercise trainings leads to remarkable gains in postural control in contrast to the application of exercise training alone.

Acknowledgements We would like to thank all our patients for taking part into the study.

Authors' Contributions Ender Ayvat: Conceptualization, data curation, formal analysis, methodology, writing — original draft, writing - review and editing. Özge Onursal Kılınç: conceptualization, formal analysis, methodology, writing-original draft. Fatma Ayvat: conceptualization, formal analysis, methodology, writing — original draft. Cevher Savcun Demirci: data curation, formal analysis, methodology, writing-original draft. Sibel Aksu Yıldırım: conceptualization, formal analysis, methodology, writing — review and editing. Oğuzhan Kurşun: conceptualization, data curation, methodology, writing — original draft. Muhammed Kılınç: conceptualization, methodology, writing—review and editing.

\section{Declarations}

Ethics Approval The study was approved by the Ethics Committee of the Hacettepe University (KA-17049) and all procedures performed in accordance with the ethical standards of the institutional and/or national research committee and with the 1964 Helsinki Declaration and its later amendments or comparable ethical standards.

Consent to Participate Before starting the experiment, all participants were informed about objectives and methods of the study and asked to sign informed consent.

Conflicts of Interest The authors declare that they have no conflict of interest.

\section{References}

1. Kandel ER, Schwartz JH, Jessell TM, Siegelbaum S, Hudspeth AJ, Mack S. Principles of neural science (4th Edition): McGraw-hill New York; 2000. 
2. Mariotti C, Fancellu R, Di Donato $S$. An overview of the patient with ataxia. J Neurol. 2005;252(5):511-8.

3. Soong B-W, Paulson HL. Spinocerebellar ataxias: an update. Curr Opin Neurol. 2007;20(4):438-46.

4. Zesiewicz TA, Wilmot G, Kuo S-H, Perlman S, Greenstein PE, Ying SH, et al. Comprehensive systematic review summary: treatment of cerebellar motor dysfunction and ataxia: report of the Guideline development, Dissemination, and Implementation Subcommittee of the American Academy of Neurology. Neurology. 2018;90(10):464-71.

5. Marquer A, Barbieri G, Pérennou D. The assessment and treatment of postural disorders in cerebellar ataxia: a systematic review. Ann Phys Rehabil Med. 2014;57(2):67-78.

6. Ilg W, Synofzik M, Brötz D, Burkard S, Giese M, Schöls L. Intensive coordinative training improves motor performance in degenerative cerebellar disease. Neurology. 2009;73(22):1823-30.

7. Omiyale O, Crowell CR, Madhavan S. Effect of Wii-based balance training on corticomotor excitability post stroke. J Mot Behav. 2015;47(3):190-200.

8. Chang Y-J, Chen S-F, Huang J-D. A Kinect-based system for physical rehabilitation: a pilot study for young adults with motor disabilities. Res Dev Disabil. 2011;32(6):2566-70.

9. Cho KH, Lee KJ, Song CH. Virtual-reality balance training with a video-game system improves dynamic balance in chronic stroke patients. Tohoku J Exp Medi. 2012;228(1):69-74.

10. Baram Y, Miller A. Virtual reality cues for improvement of gait in patients with multiple sclerosis. Neurology. 2006;66(2):178-81.

11. Bonnechère B, Jansen B, Omelina L, Van Sint JS. The use of commercial video games in rehabilitation: a systematic review. Int J Rehabil Res. 2016;39(4):277-90.

12. Synofzik M, Ilg W. Motor training in degenerative spinocerebellar disease: ataxia-specific improvements by intensive physiotherapy and exergames. BioMed Res Int. 2014;2014:583507.

13. He M, Zhang H-n, Tang Z-c, Gao S-g. Balance and coordination training for patients with genetic degenerative ataxia: a systematic review. J Neurol. 2020:1-16. [published online ahead of print, 2020 Jun 24].

14. Ilg W, Schatton C, Schicks J, Giese MA, Schöls L, Synofzik M. Video game-based coordinative training improves ataxia in children with degenerative ataxia. Neurology. 2012;79(20):2056-60.

15. Santos G, Zeigelboim DBS, Severiano M, Teive H, Liberalesso $\mathrm{P}$, Marques J, et al. Feasibility of virtual reality-based balance rehabilitation in adults with spinocerebellar ataxia: a prospective observational study. Hear Balance Commun. 2017;15(4):244-51.

16. Schatton C, Synofzik M, Fleszar Z, Giese MA, Schöls L, Ilg W. Individualized exergame training improves postural control in advanced degenerative spinocerebellar ataxia: a raterblinded, intra-individually controlled trial. Parkinsonism Relat D. 2017;39:80-4.

17. Molloy DW, Standish TI. A guide to the standardized Mini-Mental State Examination. Int Psychogeriatr. 1997;9(S1):87-94.
18. Kurtzke JF. Rating neurologic impairment in multiple sclerosis: an expanded disability status scale (EDSS). Neurology. 1983;33(11):1444-52.

19. Horak FB. Postural orientation and equilibrium: what do we need to know about neural control of balance to prevent falls? Age Ageing. 2006;35(suppl_2):ii7-11.

20. Trouillas P, Takayanagi T, Hallett M, Currier R, Subramony S, Wessel K, et al. International Cooperative Ataxia Rating Scale for pharmacological assessment of the cerebellar syndrome. J Neurol Sci. 1997;145(2):205-11.

21. Podsiadlo D, Richardson S. The timed "Up \& Go": a test of basic functional mobility for frail elderly persons. J Am Geriatr Soc. 1991;39(2):142-8.

22. Berg KO, Maki BE, Williams JI, Holliday PJ, Wood-Dauphinee SL. Clinical and laboratory measures of postural balance in an elderly population. Arch Phys Med Rehab. 1992;73(11):1073-80.

23. Clark S, Rose DJ, Fujimoto K. Generalizability of the limits of stability test in the evaluation of dynamic balance among older adults. Arch Phys Med Rehab. 1997;78(10):1078-84.

24. Franchignoni F, Horak F, Godi M, Nardone A, Giordano A. Using psychometric techniques to improve the Balance Evaluation Systems Test: the mini-BESTest. J Rehabil Med. 2010;42(4):323-31.

25. Wang R-Y, Huang F-Y, Soong B-W, Huang S-F, Yang Y-R. A randomized controlled pilot trial of game-based training in individuals with spinocerebellar ataxia type 3. Sci Rep. 2018;8(1):1-7.

26. Brooks V. The neuronal basis of motor control. New York Oxford. 1986;11:88-95.

27. Missaoui B, Thoumie P. How far do patients with sensory ataxia benefit from so-called "proprioceptive rehabilitation"? Clin Neurophysiol. 2009;39(4-5):229-33.

28. Camicioli R, Howieson D, Lehman S, Kaye J. Talking while walking: the effect of a dual task in aging and Alzheimer's disease. Neurology. 1997;48(4):955-8.

29. Fritz NE, Cheek FM, Nichols-Larsen DS. Motor-cognitive dualtask training in neurologic disorders: a systematic review. J Neurol Phys Ther. 2015;39(3):142.

30. Yogev-Seligmann G, Hausdorff JM, Giladi N. The role of executive function and attention in gait. Movement Disord. 2008;23(3):329-42.

31. Lacorte E, Bellomo G, Nuovo S, Corbo M, Vanacore N, Piscopo $\mathrm{P}$. The use of new mobile and gaming technologies for the assessment and rehabilitation of people with ataxia: a systematic review and meta-analysis. Cerebellum. 2020:1-13. [published online ahead of print, 2020 Nov 14].

Publisher's Note Springer Nature remains neutral with regard to jurisdictional claims in published maps and institutional affiliations. 